\title{
Solar Eruptions - the Effects on the Earth's Environment
}

\section{P. Brekke}

ESA Space Science Department, Goddard Space Flight Center, Mailcode 682.3, G1, Greenbelt, $M D$ 20771, USA

\begin{abstract}
The response of our space environment to the constantly changing Sun is known as "Space Weather". The Solar and Heliospheric Observatory (SOHO) has obtained significant new information about coronal mass ejections (CMEs), the source of the most severe disturbances in the Earth's environment. Most of the time space weather is of little concern in our everyday lives. However, when the space environment is disturbed by the variable outputs of the Sun, technologies that we depend on both in orbit and on the ground can be affected. The increasing deployment of radiation-, current-, and field-sensitive technological systems over the last few decades and the increasing presence of complex systems in space combine to make society more vulnerable to solar-terrestrial disturbances. Thus, our society is much more sensitive to space weather activity today compared to the last solar maximum. By observing the Sun 24 hours per day, SOHO has proved to be an important "space weather watchdog". The importance of real-time monitoring of the Sun will be pointed out and a number of enterprises affected by space weather will be discussed.
\end{abstract}

\section{Introduction}

For thousands of years people in the north marvelled at the space weather seen in the Northern Lights. But auroras never hurt a sailor or a farmer. It is only with our modern electrical, electronic and space technologies that the Sun's effects become damaging, and even personally hazardous for astronauts. The more we do in space, the more serious and potentially costly the problems will become.

The response of the space environment particularly around the Earth to the constantly changing Sun is known as 'space weather'. Most of the time space weather is of little concern in our everyday lives. However, when the space environment is disturbed by the variable outputs of the Sun, technologies that we depend on can be affected.

The increasing deployment of radiation-, current-, and field-sensitive technological systems over the last few decades and the increasing presence of complex systems in space, combine to make society more vulnerable to solarterrestrial disturbances. This has been demonstrated by the large number of problems associated with the severe magnetic storms between 1989 and 1991 and more recently during the the maximum of cycle 23 . 


\section{The Active Sun}

Space weather disturbances are generally caused by transient events in the solar atmosphere. There are two different types of events which trigger disturbances in the Earth's environment. One type is called a solar flare because the brightening of a small area on the Sun heralds its occurrence. However, not all solar flares result in geomagnetic storms, and, even more significantly, not all geomagnetic storms can be associated with solar flares. Some of the most dramatic space weather effects occur in association with eruptions of material from the solar atmosphere into interplanetary space. These eruptions are known as coronal mass ejections, or CMEs. Such eruptions are sometimes associated with flares and sometimes not and they now appear to be a primary cause of geomagnetic activity.

The emission from the two types of disturbances can be divided into two classes, electromagnetic radiation and particles which will have different effects on the Earth's environment, as discussed below.

\section{Particle Radiation}

A continuous flow of charged particles (protons and electrons) are streaming out from the Sun and is called the solar wind. Several types of solar events can cause particles with high velocities to be superimposed on this background solar wind. CMEs are believed to be caused by sudden disruptions in the Sun's magnetic field. These magnetic fields stretch and twist like titanic rubber bands until they snap. A large CME can contain 1000 million tons of matter that can reach speeds at the Earth up to $2000 \mathrm{~km} \mathrm{~s}^{-1}$, considerably greater than the normal solar wind speeds of about $400 \mathrm{~km} \mathrm{~s}^{-1}$. Thus, unlike the solar flares which emit enhanced UV/X-ray radiation, CMEs result in "clouds" of charged particles (ions and electrons). These clouds often bring with them parts of the solar magnetic field and are often named magnetic clouds. The charged particles and the magnetic fields will interact with the Earth's magnetic field when the magnetic clouds reach the Earth's orbit. This results in a disturbance of the Earth's magnetic field and the auroral particle precipitation into the atmosphere increases. The aurora is a dynamic and delicate visual manifestation of solarinduced geomagnetic storms.

One of the most dramatic effects on ground systems during geomagnetic storms is the disruption of power systems. During a geomagnetic storm the ionospheric currents, or electrojets, reach tens of thousands of amperes and produce fluctuations in the Earth's magnetic field. Electric power systems on the ground can be affected by the enhanced currents that flow in the magnetosphereionosphere system during geomagnetic disturbances. Such disturbances can induce near DC currents (Geomagnetically Induced Currents, GIC) in long power lines. For instance, during the March 13, 1989, storm, GIC's caused a complete shutdown of the Hydro-Quebec power grid resulting in a nine hour power outage. The power pools that served the entire northeastern United States came uncomfortably close to a cascading system collapse.

The enhanced particle density within the Earth's magnetic fields during a geomagnetic storm can also cause damages to satellites. Less energetic parti- 
cles contribute to a variety of spacecraft surface charging problems, especially during periods of high geomagnetic activity. In addition, energetic electrons responsible for deep dielectric charging can degrade the useful lifetime of internal components.

Under some conditions solar eruptions can also accelerate charged particles with high energies (protons and heavy particles such as helium). These highly energetic particles can penetrate to electronic components, causing bit-flips in a chain of electronic signals that may result in spurious commands (phantom commands), appearing to spacecraft systems as being sent from the ground control. In addition one can experience erroneous data from the on-board instruments. These spurious commands have caused major failures to satellite systems, even causing the craft to point away from the Earth direction. Energetic solar protons are also a radiation health hazard for astronauts on manned space flights. In addition, the radiation exposure to passengers in high-altitude aircraft is a concern.

\section{Electromagnetic Radiation}

Less than one percent of the Sun's total emitted electromagnetic radiation lies in the EUV/X-ray and radio-wave regions. Still we have a two-fold problem. First, solar activity can cause the amount of EUV/X-ray emission to be enhanced by a factor of 100 , and radio-wave emission by a factor of tens of thousands, over the normal solar output at these wavelengths. Second, it is exactly these wavelengths to which the many radar, communications, and space systems are most vulnerable.

The energetic radiation bursts from flares travel at the speed of light, and so arrive at Earth just eight minutes after leaving the flare site, well ahead of any particles or coronal material also associated with the flare. Moreover, unlike the electrons and ions of the solar wind plasma and the solar energetic particle populations, the passage of electromagnetic waves is not affected by the presence of Earth's magnetic field. The direct response of the upper atmosphere to a burst of solar flare ultraviolet and X-ray emissions is a temporary increase in ionization (as well as temperature) in the sunlit hemisphere lasting from minutes to hours and called a sudden ionospheric disturbance (SID).

In general the geomagnetic storms and increased solar ultraviolet emission heat the Earth's upper atmosphere, causing it to expand. The heated air rises, and the density at the orbit of satellites up to about $1000 \mathrm{~km}$ from the Earth increases significantly. This results in increased drag on satellites that may alter an orbit so that the satellite is temporarily "lost" to communication links. Several kilometers drop in altitude has been observed in connection with one single solar event. At times, these effects may be sufficiently severe as to cause premature re-entry of orbiting objects, such as Skylab in 1979 and Solar Maximum Mission in 1989. Unless low-Earth-orbit satellites are routinely boosted to higher orbits, they slowly fall, and eventually burn up in Earth's atmosphere.

Listed below are a variety of effects on Earth from solar particles other than those mentioned above. 
- Effects on Communication Systems: Shortwave radio communication at HF frequencies (3-30 megahertz), which is still extensively used by the military and for overseas broadcasting

- Effects on Geomagnetic Surveys: Signals used in search for natural resources (e.g. oil and gas) are significantly affected by a geomagnetic storm

- Effects on Navigation Systems: Systems such as LORAN and OMEGA are adversely affected when solar activity disrupts their radio wavelengths. It also introduce position errors and decreases the accuracy and reliability of the Global Positioning System (GPS).

- Effects of oil/gas pipelines: Space weather-induced currents similarly flow in long conductors on the ground such as oil pipelines. These currents create galvanic effects that lead to rapid corrosion at the pipeline joints if they are not properly grounded. Such corrosion requires expensive repairs or can lead to permanent damage.

\section{Space Weather Forecast}

Today our society is much more sensitive to space weather activity than was the case during the last solar maximum in 1991. An example is the possible disruption of satellites. Our society depends on satellites for weather information, communications, navigation, exploration, search and rescue, research, and defense systems. Thus, the impact of satellite system failures is more far-reaching than ever before, and the trend will almost certainly continue at an increasing rate. Furthermore, safe operation of the International Space Station depends on timely warnings of eruptions on the Sun.

It is therefore important to forecast and warn about major solar storms. The presence of two satellites located in the L1 Lagrangian point, SOHO and ACE, has definitely improved the accuracy of space weather forecasts. Two instruments on SOHO have proved to be especially valuable for continuous real-time monitoring of solar storms that affect space weather. One is the Extreme Ultraviolet Imaging Telescope (EIT) that provides images of the solar atmosphere at four wavelengths. It reveals flares and other stormy events in the atmosphere. The other is the Large Angle Spectrometric Coronagraph (LASCO) that takes images of the solar corona by blocking the light coming directly from the Sun itself with an occulter disk, creating an artificial eclipse within the instrument. It is the perfect tool for detecting CMEs heading towards the Earth.

Before SOHO was operational, only $27 \%$ of major magnetic storms (Kp index of 6 or greater) were correctly forecast, and most forecasts were false alarms. The improvement offered by SOHO is apparent in a study of 25 frontside halo CMEs seen by LASCO and EIT during 1996 and 1997 . Over $85 \%$ caused major magnetic storms and only $15 \%$ of such storms were not predicted.

\section{The 14 July 2000 Event}

A big solar explosion last month demonstrated the leading role of the SOHO spacecraft in the early-warning system for space weather. On 14 July, SOHO's 

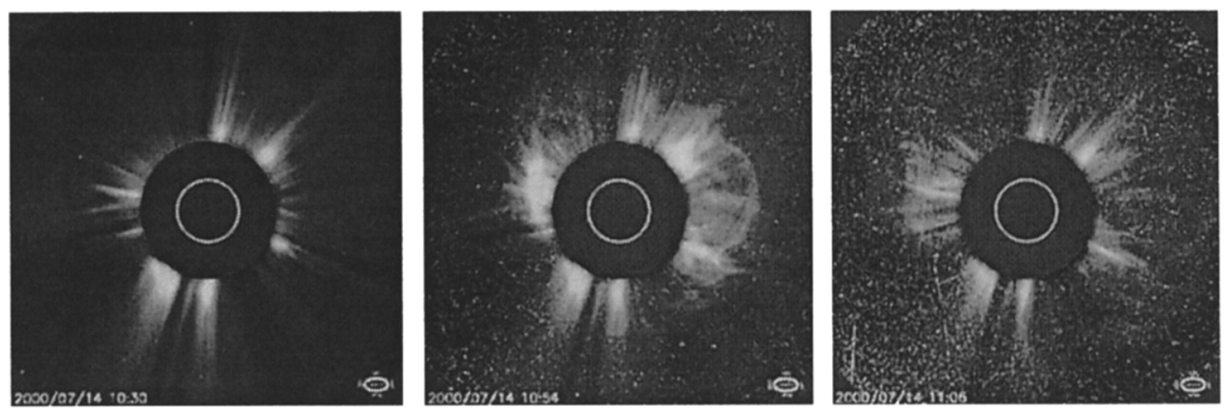

Figure 1. A full halo coronal mass ejection (CME) was recorded on July 14,2000 , by SOHO's LASCO/C2 coronagraph. The many speckles in the last two images are energetic particles bombarding SOHO's electronic detectors.

ultraviolet telescope EIT saw the bright flash of a solar flare near the center of the Sun's disk, at 10:12 Universal Time (GMT). The flare's intensity peaked at 10:24, and half an hour later SOHO's LASCO instrument detected a mass of gas racing out from the Sun (Figure 1).

Next, a burst of energetic particles from the solar explosion hit SOHO. In the imaging instruments it looked like a snowstorm that continued for some hours. Traveling more slowly than the energetic particles, the interplanetary shock wave driven by the gas of the CME arrived at SOHO a day later, at 14:19 UT on 15 July. The solar-wind instrument CELIAS on SOHO registered a jump in the wind speed from 500 to $800 \mathrm{~km} \mathrm{~s}^{-1}$, increasing to over $900 \mathrm{~km} \mathrm{~s}^{-1}$ an hour later. As the spacecraft is stationed 1.5 million kilometers out, on the sun-ward side of the Earth, the CME slammed into the Earth's magnetic field half an hour later than at SOHO, provoking auroral displays that peaked in the early hours of 16 July.

The geomagnetic activity between July 14 to July 19 produced some of the largest space weather events in this solar cycle. This was the largest geomagnetic storm observed since 1989, and one of the most intense solar proton events ever recorded. Several satellites experienced problems, and some permanent damages were reported. The storm left the Advanced Satellite for Cosmology and Astrophysics (ASCA) spinning out of control and it was considered lost. The SOHO spacecraft suffered permanent degradation of its solar panel output (losing the equivalent of 1 year of normal degradation in 24 hours). The WIND satellite experienced $25 \%$ power loss on one of the power amplifiers. Power companies reported many disturbances in their systems and one step-up transformer was significantly damaged. There were also severe blackouts of radio communications as well as degraded navigational signals.

Accurate forecasting and alerting by NOAA's Space Environment Center throughout the sequence of activity allowed users such as the electric power industry and satellite operators to be prepared for the storm. This may have reduced the damages during this particular event. 